\title{
Fine decouplings of regular differential algebraic equations
}

\author{
Roswitha März
}

\begin{abstract}
In this paper, the previously formulated conjecture concerning the existence of fine and complete decouplings of regular DAEs with tractability index $\mu$ is verified.
\end{abstract}

Keywords: differential algebraic equations, regularity, tractability index, decoupling, projectors

AMS subject classification: $34 \mathrm{~A} 09,34 \mathrm{~A} 30,34 \mathrm{~A} 12$

\section{Introduction}

Positive results obtained in [3,7] for low index cases, hae given rise to the opinion $[1$, conjecture 4.7] that an arbitrary regular differential algebraic equation (DAE)

$$
A(D x)^{\prime}+B x=q
$$

with properly stated leading term and continuous coefficients can be completely decomposed into independent, characteristic components by means of decoupling projectors, i.e., into an explicit ordinary differential equation (ODE) and assessments with included differentiations. A regular DAE according to [1] is one with a well defined tractability index $\mu$.

In this paper we will show that this conjecture turns out to be true, provided that certain subspaces of $i m D$ have continuously differentiable basis functions. This additional smoothness is closely related with the solvability of the DAE itself and can lead to differentiability requirements for certain parts of the coefficients $A, D$ and $B$. However, the coefficients of the DAE are not differentiated, and we do without derivative arrays.

Fine decouplings are convenient for the further analysis of DAEs.

The concept of the tractability index has been designed for DAEs with (almost) only continuous coefficients and is related with a number of constant rank conditions, which are essential here. Points with rank changes have to be treated separately as critical points, as already pointed out in [2].

For a presentation of results on DAEs with smooth coefficients and on reduction techniques using transformations we refer to the comprehensive overview [5]. In contrast to those results the decoupled system is noted in the original variables here. In case of a fine decoupling, the explicit ODE is uniquely determined, which is e.g. important for qualitative investigations of the flow.

A constant coefficient DAE is regular with tractability index $\mu$ if the corresponding matrix pencil is regular with Kronecker index $\mu$. A variable coefficient DAE that can be reduced to strong standard canonical form (SSCN) (cf. [6]) by scaling and a transformation of the unknown function is regular with a tractability index that equals the nilpotency index in the SSCN ([2]).

Below we will realize a general regular DAE (1.1) to be equivalent to a DAE in standard canonical 
form (SCF) (cf. [4]), supposed that the mentioned smoothness for the fine decoupling is given. The aim of this paper is just the verification of fine decoupling. For more general discussions on DAE concepts we refer to [5], but also to [2].

The paper is organized as follows:

Section 2 describes the necessary material from [1] and [2]. Section 3 deals with index-2 DAEs as a case study. Fine and complete decouplings are constructed in the Sections 4 and 5, respectively. Section 5 contains the SCF. The concluding remark follows as Section 6. The Appendix contains the proof of Lemma 4.2, which gives the basic rules for the construction of fine decouplings.

Let us mention here that a linear DAE in the usual form

$$
E x^{\prime}+F x=q
$$

with continuous coefficients $E, F$ may be rewritten as (1.1) with properly stated leading term supposed $E$ has constant rank and its nullspace is spanned by continuously differentiable basis functions. Then one may decompose $E=\mathcal{U}^{*}\left(\begin{array}{cc}\Sigma & 0 \\ 0 & 0\end{array}\right) V, \Sigma$ is nonsingular, $\mathcal{U}, \Sigma$ are continuous, $V$ is continuously differentiable. For instance, with $A=\mathcal{U}^{*}\left(\begin{array}{c}\Sigma \\ 0\end{array}\right), D=\left(\begin{array}{ll}I & 0\end{array}\right) V$ and $B=F-A D^{\prime}$ we obtain a DAE of the form (1.1). Another possible version is, by means of the Moore-Penrose inverse $E^{+}$,

$$
E\left(E^{+} E x\right)^{\prime}+\left(F-E\left(E^{+} E\right)^{\prime}\right) x=q .
$$

If $E$ itself is continuously differentiable, we may also use

$$
E E^{+}(E x)^{\prime}+\left(F-E E^{+} E^{\prime}\right) x=q .
$$

By means of those reformulations the results obtained for (1.1) apply to DAEs of the form (1.2), too.

\section{Fundamentals}

We deal with equations

$$
A(t)(D(t) x(t))^{\prime}+B(t) x(t)=q(t), \quad t \in \mathcal{I},
$$

with continuous coefficients $A(t) \in L\left(\mathbb{R}^{n}, \mathbb{R}^{m}\right), D(t) \in L\left(\mathbb{R}^{m}, \mathbb{R}^{n}\right), B(t) \in L\left(\mathbb{R}^{m}\right), t \in \mathcal{I}, \mathcal{I} \subseteq \mathbb{R}$ an interval, and with properly stated leading term.

The leading term in (2.1) is stated properly if the matrix functions $A$ and $D$ are well matched in the sense that the decomposition

$$
\operatorname{ker} A(t) \oplus i m D(t)=\mathbb{R}^{n}, \quad t \in \mathcal{I},
$$

is valid and both subspaces $\operatorname{ker} A$ and $i m D$ are $C^{1}$, i.e., they are spanned by $C^{1}$ basis functions. Let $R(t)$ be given by

$$
R(t)^{2}=R(t), \quad i m R(t)=i m D(t), \operatorname{ker} R(t)=\operatorname{ker} A(t) .
$$

Then, $R$ is a continuously differentiable projector function. Additionally, we use a function $D^{-} \in$ $C\left(\mathcal{I}, L\left(\mathbb{R}^{n}, \mathbb{R}^{m}\right)\right)$ such that the conditions

$$
D(t) D(t)^{-} D(t)=D(t), \quad D(t)^{-} D(t) D(t)^{-}=D(t)^{-}, \quad D(t) D(t)^{-}=R(t), t \in \mathcal{I},
$$


are satisfied. $D^{-}$is pointwise a generalized inverse of $D$. By this, $D^{-}$is not uniquely determined. $D^{-} D=: P_{0}$ is a continuous projector function with $\operatorname{ker} P_{0}=\operatorname{ker} D=\operatorname{ker} A D$.

A solution of (2.1) is a function $x: \mathcal{I} \subseteq \mathbb{R} \rightarrow \mathbb{R}^{m}$, which belongs to the function space $C_{D}^{1}$ and satisfies (2.1) pointwise. $C_{D}^{1}$ is the short notation for

$$
C_{D}^{1}\left(\mathcal{I}, \mathbb{R}^{m}\right):=\left\{x \in C\left(\mathcal{I}, \mathbb{R}^{m}\right): D x \in C^{1}\left(\mathcal{I}, \mathbb{R}^{n}\right)\right\}
$$

Next, we recall from [1] the special sequence of matrix functions and subspaces to be used for index characterization and system decoupling, namely

$$
G_{0}:=A D, \quad N_{0}:=\operatorname{ker} G_{0}, \quad B_{0}:=B, \quad Q_{0}:=I-P_{0},
$$

and for $i \geq 0$,

$$
\begin{aligned}
G_{i+1} & :=G_{i}+B_{i} Q_{i}, \quad N_{i+1}:=\operatorname{ker}_{i+1}, \\
Q_{i+1}^{2} & =Q_{i+1}, \quad i m Q_{i+1}=N_{i+1}, P_{i+1}:=I-Q_{i+1}, \\
B_{i+1} & :=B_{i} P_{i}-G_{i+1} D^{-}\left(D P_{0} \cdots P_{i+1} D^{-}\right)^{\prime} D P_{0} \cdots P_{i} .
\end{aligned}
$$

Here, if the argument $t$ is dropped, the given relations are meant pointwise. The matrices $Q_{i}(t)$ and $P_{i}(t)$ represent complementary projectors acting in $\mathbb{R}^{m} ; G_{i}(t)$ and $B_{i}(t)$ are $m \times m$, and the nullspace $N_{i}(t)$ is a subspace in $\mathbb{R}^{m}$. When using (2.6) one has to take care of the existence of the involved derivative.

Definition 2.1 ([1]) The sequence (2.3) - (2.6) is said to be admissible up to $k \in \mathbb{I}$ resp. the projector functions $Q_{0}, \ldots, Q_{k}$ are admissible if

(a) $\operatorname{rank}_{i}(t)=r_{i}, t \in \mathcal{I}, i=0, \ldots, k$,

(b) $N_{0} \oplus \cdots \oplus N_{i-1} \subseteq k e r Q_{i}, i=1, \ldots, k$,

(c) $Q_{i} \in C\left(\mathcal{I}, L\left(\mathbb{R}^{m}\right)\right), D P_{0} \cdots P_{i} D^{-} \in C^{1}\left(\mathcal{I}, L\left(\mathbb{R}^{n}\right)\right), i=0, \ldots, k$.

Definition 2.2 Equation (2.1) is called a regular DAE or, more precisely, a regular DAE with tractability index $\mu$ if there is a sequence (2.3)-(2.6) that is admissible up to $\mu \in \mathbb{N}$, and such that $r_{\mu-1}<r_{\mu}=m$.

For a regular DAE, the sequence (2.3) - (2.6) could be continued formally by $G_{\mu+j}=G_{\mu}$, hence Definition 2.2 coincides with the definition first given in [2].

Regularity of a DAE (2.1) as well as the index $\mu$ are independent of the choice of the admissible projectors. They are also invariant with respect to regular transformations of the unknown function and refactorizations of the leading term (cf [1], [2]).

Recall that property (b) of admissible projector functions makes products $P_{0} P_{1} \cdots P_{i}$, $P_{0} P_{1} \cdots P_{i-1} Q_{i}, D P_{0} P_{1} \cdots P_{i} D^{-}$, and so on, become projector functions, too. A regular DAE (2.1) may be scaled by $G_{\mu}^{-1}$ to (cf. [2])

$$
\begin{aligned}
P_{\mu-1} & \cdots P_{0} D^{-}(D x)^{\prime}+G_{\mu}^{-1} B P_{0} \cdots P_{\mu-1} x+\sum_{j=0}^{\mu-1} Q_{j} x \\
& +\sum_{i=1}^{\mu-1} \sum_{i=1}^{i} P_{\mu-1} \cdots P_{j} D^{-}\left(D P_{0} \cdots P_{j} D^{-}\right)^{\prime} D P_{0} \cdots P_{i-1} Q_{i} x=G_{\mu}^{-1} q .
\end{aligned}
$$


The scaled version (2.7) of the regular DAE (2.1) shows more inherent structure. Via

$$
\begin{aligned}
& u=D P_{0} \cdots P_{\mu-1} x, v_{0}=Q_{0} x, v_{i}=P_{0} \cdots P_{i-1} Q_{i} x, i=1, \ldots, \mu-1, \\
& x=D^{-} u+v_{0}+\ldots+v_{\mu-1},
\end{aligned}
$$

we obtain (cf. [1], [2] for quite long technical calculations) an equivalent, decoupled system

$$
\begin{gathered}
u^{\prime}-\left(D P_{0} \cdots P_{\mu-1} D^{-}\right)^{\prime} u+D P_{0} \cdots P_{\mu-1} G_{\mu}^{-1} B D^{-} u=D P_{0} \cdots P_{\mu-1} G_{\mu}^{-1} q, \\
v_{\mu-1}=-\mathcal{K}_{\mu-1} D^{-} u+\mathcal{L}_{\mu-1} q, \\
v_{k}=-\mathcal{K}_{k} D^{-} u+\sum_{j=k+1}^{\mu-1} \mathcal{N}_{k j}\left(D v_{j}\right)^{\prime}+\sum_{j=k+2}^{\mu-1} \mathcal{M}_{k j} v_{j}+\mathcal{L}_{k} q, k=0, \ldots, \mu-2,
\end{gathered}
$$

with continuous coefficients $\mathcal{K}_{k}, \mathcal{N}_{k j}, \mathcal{M}_{k j}, \mathcal{L}_{k}$ described in detail in [1]. Here our special interest is directed to the coupling coefficients $\mathcal{K}_{0}, \ldots, \mathcal{K}_{\mu-1}$, i.e.,

$$
\begin{aligned}
\mathcal{K}_{0}= & Q_{0} P_{1} \cdots P_{\mu-1} G_{\mu}^{-1}\left\{B_{0}+G_{0} D^{-}\left(D P_{0} \cdots P_{\mu-1} D^{-}\right)^{\prime} D\right\} P_{0} \cdots P_{\mu-1}, \\
\mathcal{K}_{k}= & P_{0} \cdots P_{k-1} Q_{k} P_{k+1} \cdots P_{\mu-1} G_{\mu}^{-1}\left\{B_{0}+G_{k} D^{-}\left(D P_{0} \cdots P_{\mu-1} D^{-}\right)^{\prime} D\right\} P_{0} \cdots P_{\mu-1} \\
\quad k=1, \ldots, \mu-2 & \\
\mathcal{K}_{\mu-1}= & P_{0} \cdots P_{\mu-2} Q_{\mu-1} G_{\mu}^{-1} B_{0} P_{0} \cdots P_{\mu-1} .
\end{aligned}
$$

Definition 2.3 ([1]) For a regular DAE with tractability index $\mu$, the admissible projector functions $Q_{0}, \ldots, Q_{\mu-1}$ provide

(i) a fine decoupling if $\mathcal{K}_{i}=0, i=1, \ldots, \mu-1$,

(ii) a complete decoupling if, additionally, $\mathcal{K}_{0}=0$.

Let $S_{\text {can } \mu}(t) \subset \mathbb{R}^{m}$ denote the geometric locus of the solution values $x(t)$ of the homogeneous DAE (2.1) with $q=0$, i.e.,

$$
S_{c a n \mu}(t):=\left\{x(t): x \in C_{D}^{1}, A(D x)^{\prime}+B x=0\right\}, t \in \mathcal{I} .
$$

Introduce further $N_{c a n \mu}:=N_{0} \oplus \cdots \oplus N_{\mu-1}$. Both, $N_{\text {can } \mu}$ and $S_{\text {can } \mu}$ are independent of the choice of admissible projectors $Q_{0}, \ldots, Q_{\mu-1}$ (cf. [1]). If admissible $Q_{0}, \ldots, Q_{\mu-1}$ provide a fine decoupling of the regular DAE (2.1), then the decomposition

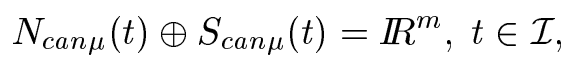

holds true, and $\Pi_{c a n \mu}=\left(I-\mathcal{K}_{0}\right) P_{0} \cdots P_{\mu-1}$ is exactly the projector function that realizes this decomposition ([1, Theorem 4.4] and Theorem 4.3 below).

Since $N_{c a n \mu}(t)$ has constant dimension $\mu m-\sum_{i=0}^{\mu-1} r_{i}, S_{c a n \mu}(t)$ must have constant dimension $d:=(1-\mu) m+\sum_{i=0}^{\mu-1} r_{i}$.

In [1] it was conjectured that fine resp. complete decouplings do exist. Here we will show this conjecture to become true supposed that there is some more smoothness in parts of the coefficients $A, D, B$ than the poor continuity we started with. We explain the situation for the case of $\mu=2$. 


\section{The known index-2 case}

For an index-2 regular DAE (2.1) the general decoupled system reads in detail

$$
\begin{gathered}
u^{\prime}-\left(D P_{0} P_{1} D^{-}\right)^{\prime} u+D P_{0} P_{1} G_{2}^{-1} B D^{-} u=D P_{0} P_{1} G_{2}^{-1} q, \\
v_{1}=-\mathcal{K}_{1} D^{-} u+P_{0} Q_{1} G_{2}^{-1} q, \\
v_{0}=-\mathcal{K}_{0} D^{-} u+Q_{0} Q_{1} D^{-}\left(D v_{1}\right)^{\prime}+Q_{0} P_{1} G_{2}^{-1} q,
\end{gathered}
$$

with $\mathcal{K}_{1}=P_{0} Q_{1} G_{2}^{-1} B_{0} P_{0} P_{1}$ and

$$
\begin{aligned}
\mathcal{K}_{0} & =Q_{0} P_{1} G_{2}^{-1}\left(B_{0}+G_{0} D^{-}\left(D P_{0} P_{1} D^{-}\right)^{\prime} D\right) P_{0} P_{1} \\
& =\left(Q_{0} P_{1} G_{2}^{-1} B_{0}-Q_{0} Q_{1} D^{-}\left(D P_{0} P_{1} D^{-}\right)^{\prime} D\right) P_{0} P_{1} \\
& =\left(Q_{0} P_{1} G_{2}^{-1} B_{0}+Q_{0} Q_{1} D^{-}\left(D P_{0} Q_{1} D^{-}\right)^{\prime} D\right) P_{0} P_{1} .
\end{aligned}
$$

Computing

$$
\begin{aligned}
Q_{1} G_{2}^{-1} B_{0} P_{0} Q_{1} & =Q_{1} G_{2}^{-1}\left(B_{1}+G_{1} D^{-}\left(D P_{0} P_{1} D^{-}\right)^{\prime} D P_{0}\right) Q_{1} \\
& =Q_{1} G_{2}^{-1} B_{1} Q_{1}=Q_{1}
\end{aligned}
$$

we learn that $\bar{Q}_{1}:=Q_{1} G_{2}^{-1} B_{0}=Q_{1} G_{2}^{-1} B_{0} P_{0}=Q_{1} G_{2}^{-1} B_{1}$ is a further continuous projector function onto $N_{1}$ satisfying the condition $N_{0} \subseteq \operatorname{ker} \bar{Q}_{1}$. However, for admissibility we have to meet the condition

$$
D P_{0} Q_{1} G_{2}^{-1} B_{0} D^{-} \in C^{1}\left(\mathcal{I}, L\left(\mathbb{R}^{n}\right)\right) .
$$

If this condition is given, the new sequence $\bar{Q}_{0}:=Q_{0}, \bar{Q}_{1}:=Q_{1} G_{2}^{-1} B_{0} P_{0}$ provides

$$
\begin{aligned}
\bar{G}_{1}=G_{1}, \bar{G}_{2} & =G_{1}+\left(B_{0} P_{0}-G_{1} D^{-}\left(D P_{0} \bar{P}_{1} D^{-}\right)^{\prime} D P_{0}\right) \bar{Q}_{1} \\
& =G_{2}\left(I+\bar{Q}_{1} P_{1}+Q_{0} Q_{1} D^{-}\left(D P_{0} \bar{P}_{1} D^{-}\right)^{\prime} D P_{0} \bar{Q}_{1}\right)=: G_{2} Z_{2}, \\
\bar{Q}_{1} \bar{G}_{2}^{-1} B_{0} P_{0} & =\bar{Q}_{1} Z_{2}^{-1} G_{2}^{-1} B_{0} P_{0}=\bar{Q}_{1}\left(I-\bar{Q}_{1} P_{1}\right) G_{2}^{-1} B_{0} P_{0}=Q_{1} G_{2}^{-1} B_{0} P_{0}=\bar{Q}_{1},
\end{aligned}
$$

and thus $\overline{\mathcal{K}}_{1}=\bar{P}_{0} \bar{Q}_{1} \bar{G}_{2}^{-1} B_{0} \bar{P}_{0} \bar{P}_{1}=\bar{P}_{0} \bar{Q}_{1} \bar{P}_{1}=0$.

It should be stressed that condition (3.4) is quite natural with respect to solvability. Put $q=0$ and look at the equations (3.2) and (3.3). For obtaining $D v_{1} \in C^{1}\left(\mathcal{I}, \mathbb{R}^{n}\right)$ and further, with $x=D^{-} u+v_{0}+v_{1}, \quad D x \in C^{1}\left(\mathcal{I}, \mathbb{R}^{n}\right)$, we need (3.4). Namely, (3.4) leads to $D \mathcal{K}_{1} D^{-} u=$ $D P_{0} Q_{1} G_{2}^{-1} B_{0} P_{0} P_{1} D^{-} u=D P_{0} Q_{1} G_{2}^{-1} B_{0} D^{-} u \in C^{1}\left(\mathcal{I}, \mathbb{R}^{n}\right)$.

Example 3.1 ([1, Example 4.2])

For the DAE

$$
\begin{array}{ll}
x_{1}^{\prime}(t) & =q_{1}(t), \\
x_{2}^{\prime}(t)-x_{3}(t) & =q_{2}(t), \\
2 \alpha(t) x_{1}(t)+x_{2}(t) & =q_{3}(t),
\end{array}
$$

we realize immediately what the solutions in case of $q=0$ look like $: x_{1}(t) \equiv x_{10}, x_{2}(t) \equiv-2 \alpha(t) x_{10}$, however, to compute $x_{3}(t)=-2 \alpha^{\prime}(t) x_{10}$ if $x_{10} \neq 0$, the coefficient $\alpha$ necessarily has to be $C^{1}$.

Written as (2.1) with

$$
A=\left(\begin{array}{ll}
1 & 0 \\
0 & 1 \\
0 & 0
\end{array}\right), \quad D=\left(\begin{array}{ccc}
1 & 0 & 0 \\
0 & 1 & 0
\end{array}\right), B=\left(\begin{array}{ccc}
0 & 0 & 0 \\
0 & 0 & -1 \\
2 \alpha & 1 & 0
\end{array}\right),
$$


this DAE is regular with tractabiliy index 2. The projectors $Q_{0}=\left(\begin{array}{lll}0 & 0 & 0 \\ 0 & 0 & 0 \\ 0 & 0 & 1\end{array}\right), Q_{1}=\left(\begin{array}{lll}0 & 0 & 0 \\ 0 & 1 & 0 \\ 0 & 1 & 0\end{array}\right)$ are admissible and yield a decoupled system with

$$
\begin{gathered}
\mathcal{K}_{1}=\left(\begin{array}{ccc}
0 & 0 & 0 \\
2 \alpha & 0 & 0 \\
0 & 0 & 0
\end{array}\right), D P_{0} Q_{1} G_{2}^{-1} B_{0} D^{-}=\left(\begin{array}{cc}
0 & 0 \\
2 \alpha & 0
\end{array}\right), \\
\bar{Q}_{1}:=Q_{1} G_{2}^{-1} B_{0} P_{0}=\left(\begin{array}{ccc}
0 & 0 & 0 \\
2 \alpha & 1 & 0 \\
2 \alpha & 1 & 0
\end{array}\right), D P_{0} \bar{P}_{1} D^{-}=\left(\begin{array}{cc}
1 & 0 \\
-2 \alpha & 0
\end{array}\right) .
\end{gathered}
$$

Remark: Concerning the index-2 case, condition (3.4) is incorporated into the definition of index-2 tractable DAEs given in [3] at the very beginning by means of the demand for the subspaces $D N_{1}$ and $D S_{1}, S_{1}:=\left\{z \in \mathbb{R}^{m}: B z \in i m G_{1}\right\}$, to be spanned by $C^{1}$ functions. In our example these subspaces are

$$
D N_{1}=\operatorname{span}\left(\begin{array}{l}
0 \\
1
\end{array}\right), D S_{1}=\operatorname{span}\left(\begin{array}{c}
1 \\
-2 \alpha
\end{array}\right)
$$

We would like to point out that these smoothness conditions are related to an appropriate further decomposition of the $C^{1}$ subspace $i m D$. There is no general need for the original coefficients $A, D, B$ to be continuously differentiable.

We finish this section on the case $\mu=2$ by noting that, given a fine decoupling sequence $Q_{0}, Q_{1}$, the projector functions

$$
\bar{Q}_{0}:=Q_{0} P_{1} G_{2}^{-1} B_{0}+Q_{0} Q_{1} D^{-}\left(D P_{0} Q_{1} D^{-}\right)^{\prime}, \bar{Q}_{1}:=\left(I-\bar{Q}_{0} P_{0}\right) Q_{1}=Q_{1}
$$

are proved to generate a complete decoupling $([3])$.

\section{Construction of fine decouplings}

Given a regular DAE (2.1) with tractability index $\mu$ and an admissible sequence $Q_{0}, \ldots, Q_{\mu-1}$. Rewrite the coupling coefficients $(2.14)-(2.15)$ as

$$
\begin{aligned}
\mathcal{K}_{0} & =Q_{0 *} P_{0} \cdots P_{\mu-1} \\
\mathcal{K}_{k} & =P_{0} \cdots P_{k-1} Q_{k *} P_{0} \cdots P_{\mu-1}, k=1, \ldots, \mu-2, \\
\mathcal{K}_{\mu-1} & =P_{0} P_{\mu-2} Q_{\mu-1 *} P_{0} \cdots P_{\mu-1}
\end{aligned}
$$

with

$$
\begin{aligned}
Q_{0 *} & :=Q_{0} P_{1} \cdots P_{\mu-1} G_{\mu}^{-1}\left\{B_{0}+G_{0} D^{-}\left(D P_{0} \cdots P_{\mu-1} D^{-}\right)^{\prime} D\right\}, \\
Q_{k *} & :=Q_{k} P_{k+1} \cdots P_{\mu-1} G_{\mu}^{-1}\left\{B_{k}+G_{k} D^{-}\left(D P_{0} \cdots P_{\mu-1} D^{-}\right)^{\prime} D P_{0} \cdots P_{k-1}\right\}, \\
k=1, \ldots, \mu-2, & \\
Q_{\mu-1 *} & :=Q_{\mu-1} G_{\mu}^{-1} B_{\mu-1} .
\end{aligned}
$$

Remark: Here, $Q_{k *}, k=1, \ldots, \mu-2$, are slightly different from those introduced in [1, Section 3], and do not lead to the desired results. 
We will show that, by a smart choice of admissible projector functions, the coefficients $\mathcal{K}_{k}, k=$ $1, \ldots, \mu-1$, disappear. Since $\operatorname{ker} P_{0} \cdots P_{k-1}=N_{0} \oplus \cdots \oplus N_{k-1} \subseteq \operatorname{ker} Q_{k}$, the coupling coefficient $\mathcal{K}_{k}$ vanishes only if its factor $Q_{k *} P_{0} \cdots P_{\mu-1}$ does so, $k=1, \ldots, \mu-1$. So we restrict our interest to these expressions.

\section{Lemma 4.1}

(i) $Q_{i *} \in C\left(\mathcal{I}, L\left(\mathbb{R}^{m}\right)\right)$ is a projector function with $i m Q_{i *}=N_{i}, i=0, \ldots, \mu-1$.

(ii) For $i=0, \ldots, \mu-1$ it holds that $N_{0} \oplus \cdots \oplus N_{i-1} \subseteq \operatorname{ker} Q_{i *}$.

Proof: We show that $Q_{i *} Q_{i}=Q_{i}$. Then, the other assertions follow from the construction. Compute

$$
Q_{0 *} Q_{0}=Q_{0} P_{1} \cdots P_{\mu-1} G_{\mu}^{-1} B_{0} Q_{0}=Q_{0} P_{1} \cdots P_{\mu-1} G_{\mu}^{-1} G_{\mu} Q_{0}=Q_{0} P_{1} \cdots P_{\mu-1} Q_{0}=Q_{0},
$$

and for $k=1, \ldots, \mu-2$

$$
\begin{aligned}
Q_{k *} Q_{k}= & Q_{k} P_{k+1} \cdots P_{\mu-1} G_{\mu}^{-1} B_{k} Q_{k}+ \\
& \quad+Q_{k} P_{k+1} \cdots P_{\mu-1} G_{\mu}^{-1} G_{k} D^{-}\left(D P_{0} \cdots P_{\mu-1} D^{-}\right)^{\prime} D P_{0} \cdots P_{\mu-1} Q_{k} \\
= & Q_{k} P_{k+1} \cdots P_{\mu-1} G_{\mu}^{-1} G_{\mu} Q_{k}+ \\
& \quad+Q_{k} P_{k+1} \cdots P_{\mu-1} G_{\mu}^{-1} G_{\mu} P_{\mu-1} \cdots P_{k} D^{-}\left(D P_{0} \cdots P_{\mu-1} D^{-}\right)^{\prime} D P_{0} \cdots P_{k-1} Q_{k} \\
= & Q_{k}+Q_{k} P_{k+1} \cdots P_{\mu-1} P_{k} D^{-}\left(D P_{0} \cdots P_{\mu-1} D^{-}\right)^{\prime} D P_{0} \cdots P_{k-1} Q_{k} D^{-} D \\
= & Q_{k}-Q_{k} P_{k+1} \cdots P_{\mu-1} P_{k} P_{0} \cdots P_{\mu-1} D^{-}\left(D P_{0} \cdots P_{k-1} Q_{k} D^{-}\right)^{\prime} D \\
= & Q_{k}, \\
Q_{\mu-1 *} Q_{\mu-1}= & Q_{\mu-1} G_{\mu}^{-1} B_{\mu-1}=Q_{\mu-1} G_{\mu}^{-1} G_{\mu} Q_{\mu-1}=Q_{\mu-1} .
\end{aligned}
$$

For any two admissible projector function sequences $Q_{0}, \ldots, Q_{\mu-1}$ and $\bar{Q}_{0}, \ldots, \bar{Q}_{\mu-1}$ the corresponding matrix functions are related by

$$
\begin{gathered}
\bar{G}_{1}=G_{1} Z_{1}, Z_{1}:=I+Q_{0} \bar{Q}_{0} P_{0}, \\
\bar{G}_{i+1}=G_{i+1} Z_{i+1}, Z_{i+1}:=\left(I+Q_{i} \bar{Q}_{i} P_{i}+\sum_{j=0}^{i-1} Q_{j} Z_{i j} \bar{Q}_{i}\right) Z_{i}, \\
i=1, \ldots, \mu-1 .
\end{gathered}
$$

The coefficients $Z_{i j}$ are continuous, their detailed form does not matter here. For the proof of (4.7), (4.8) we refer to [1].

Given the admissible projector functions $Q_{0}, \ldots, Q_{\mu-1}$ we now construct new ones $\bar{Q}_{0}, \ldots, \bar{Q}_{\mu-1}$ in the following way. We fix $k \in\{1, \ldots, \mu-1\}$ and choose $\bar{Q}_{i}:=Q_{i}, i=0, \ldots, k-1$.. Then we take a new continuous $\bar{Q}_{k}$ such that $\bar{N}_{0} \oplus \cdots \oplus \bar{N}_{k-1}=N_{0} \oplus \cdots \oplus N_{k-1} \subseteq \operatorname{ker} \bar{Q}_{k} . D P_{0} \cdots P_{k-1} \bar{P}_{k} D^{-}$ is assumed to be continuously differentiable. Further we choose $\bar{Q}_{i}:=Z_{i}^{-1} Q_{i} Z_{i}=Z_{i}^{-1} Q_{i}$, $i=$ $k+1, \ldots, \mu-1$, if $k \leq \mu-2$.

Thereby, we have for $i=k+1, \ldots, \mu-1$,

$$
\begin{aligned}
\bar{Q}_{i} \bar{Q}_{j} & =Z_{i}^{-1} Q_{i} Q_{j}=0, \text { for } j=0, \ldots, k-1, \\
\bar{Q}_{i} \bar{Q}_{k} & =Z_{i}^{-1} Q_{i} Q_{k} \bar{Q}_{k}=0 \\
\bar{Q}_{i} \bar{Q}_{j} & =Z_{i}^{-1} Q_{i} Z_{i}^{-1} Q_{j}=Z_{i}^{-1} Q_{i} Q_{j}=0, \text { for } j=k+1, \ldots, i-1,
\end{aligned}
$$


and, additionally, $\bar{Q}_{i} P_{i}=Z_{i}^{-1} Q_{i} P_{i}=0$, so that the factors $Z_{1}, \ldots, Z_{\mu}$ (cf. (4.7), (4.8)) simplify to $Z_{i}=I, i=1, \ldots, k$,

$$
\begin{aligned}
Z_{k+1} & =I+\bar{Q}_{k} P_{k}+\sum_{j=0}^{k-1} Q_{j} Z_{k j} \bar{Q}_{k} \\
Z_{i+1} & =\left(I+\sum_{j=0}^{i-1} Q_{j} Z_{i j} \bar{Q}_{i}\right) Z_{i}, \quad i=k+1, \ldots, \mu-1 .
\end{aligned}
$$

Compute

$$
Z_{k+1}^{-1}=I-\bar{Q}_{k} P_{k}-\sum_{j=0}^{k-1} Q_{j} Z_{k j} Q_{k}
$$

and, for $i=k+1, \ldots, \mu-1$,

$$
Z_{i+1}^{-1}=Z_{i}^{-1}=\left(I-\sum_{j=0}^{i-1} Q_{j} Z_{i j} Q_{i}\right), \quad Z_{i} Z_{i+1}^{-1}=I-\sum_{j=0}^{i-1} Q_{j} Z_{i j} Q_{i}
$$

and, moreover,

$$
\begin{aligned}
\bar{P}_{0} \cdots \bar{P}_{i} & =P_{0} \cdots P_{k-1} \bar{P}_{k} \bar{P}_{k+1} \cdots \bar{P}_{i}=P_{0} \cdots P_{k-1}\left(P_{k}+Q_{k}\right) \bar{P}_{k} \cdots \bar{P}_{i} \\
& =P_{0} \cdots P_{k} Z_{k+1}^{-1} P_{k+1} Z_{k+1} \cdots Z_{i}^{-1} P_{i} Z_{i}+P_{0} P_{k-1} Q_{k} \bar{P}_{k} \cdots \bar{P}_{i} \\
& =P_{0} \cdots P_{i}+P_{0} \cdots P_{k-1} Q_{k} \bar{P}_{k} \bar{P}_{k+1} \cdots \bar{P}_{i} \\
& =P_{0} \cdots P_{i}-P_{0} \cdots P_{k-1} \bar{Q}_{k} P_{k} \cdots P_{i}, \\
D \bar{P}_{0} \cdots \bar{P}_{i} D^{-} & =D P_{0} \cdots P_{i} D^{-}-D P_{0} \cdots P_{k-1} \bar{Q}_{k} D^{-} \cdot D P_{0} \cdots P_{i} D^{-} .
\end{aligned}
$$

Since $D P_{0} \cdots P_{k-1} \bar{Q}_{k} D^{-}=D P_{0} \cdots P_{k-1} D^{-}-D P_{0} \cdots P_{k-1} \bar{P}_{k} D^{-}$is continuously differentiable, so are the $D \bar{P}_{0} \ldots \bar{P}_{i} D^{-}$, i.e., our new $\bar{Q}_{0}, \ldots, \bar{Q}_{\mu-1}$ are admissible.

Lemma 4.2 For a fixed $k \in\{1, \ldots, \mu-2\}$, let $Q_{i *} P_{0} \cdots P_{\mu-1}=0, i=k+1, \ldots, \mu-1$. Let $\bar{Q}_{j}=Q_{j}, j=0, \ldots, k-1, \bar{Q}_{k}^{2}=\bar{Q}_{k}, \operatorname{ker} \bar{Q}_{k} \supseteq N_{0} \oplus \cdots \oplus N_{k-1}, i m \bar{Q}_{k}=N_{k}, D P_{0} \cdots P_{k-1} \bar{Q}_{k} D^{-} \in$ $C^{1}\left(\mathcal{I}, L\left(\mathbb{R}^{n}\right)\right), \bar{Q}_{j}:=Z_{j}^{-1} Q_{j} Z_{j}, j=k+1, \ldots \mu-1$. Then, $\bar{Q}_{0}, \ldots, \bar{Q}_{\mu-1}$ are admissible and the relations

$$
\begin{aligned}
\bar{Q}_{i *} \bar{P}_{0} \cdots \bar{P}_{\mu-1} & =0, \quad i=k+1, \ldots, \mu-1 \\
\bar{Q}_{k *} \bar{P}_{0} \cdots \bar{P}_{\mu-1} & =\left(Q_{k *}-\bar{Q}_{k}\right) P_{0} \cdots P_{\mu-1}
\end{aligned}
$$

become true.

Proof: It remains to show (4.12),(4.12). This is done by longer but straightforward technical computations in the Appendix below.

As discussed e.g. in [1], we may a priori choose $Q_{\mu-1}$ onto $N_{\mu-1}$ along $S_{\mu-1}:=\left\{z \in R^{m}: B_{\mu-1} z \in\right.$ $\left.i m G_{\mu-1}\right\}=\left\{z \in \mathbb{R}^{m}: B_{0} P_{0} \cdots P_{\mu-2} z \in i m G_{\mu-1}\right\}=\left\{z \in \mathbb{R}^{m}: B_{0} z \in i m G_{\mu-1}\right\}$, since, in the index $\mu$ case, the decomposition $N_{\mu-1} \oplus S_{\mu-1}=\mathbb{R}^{m}$ is valid.

However, this yields

$$
\begin{aligned}
Q_{\mu-1} & =Q_{\mu-1} G_{\mu}^{-1} B_{\mu-1} \\
\text { i.e., } Q_{\mu-1} & =Q_{\mu-1 *}, Q_{\mu-1 *} P_{0} \cdots P_{\mu-1}=0 .
\end{aligned}
$$


Let us stress again (cf. $\S 3$ ) that an additional smoothness condition for the original DAE coefficient may be attached to the condition $D P_{0} \cdots P_{\mu-2} P_{\mu-1 *} D^{-} \in C^{1}\left(\mathcal{I}, L\left(\mathbb{R}^{n}\right)\right)$ ensuring admissibility.

Lemma 4.2 indicates how to proceed further. Namely, having (4.13), i.e., $K_{\mu-1}=0$, we put $\bar{Q}_{\mu-2}=Q_{\mu-2 *}$ and obtain $\bar{K}_{\mu-1}=0, \bar{K}_{\mu-2}=0$, and so on. In each step, at least one further coupling coefficient disappears.

Theorem 4.3 Let the DAE (2.1) be regular with tractability index $\mu$, and let its coefficients be sufficiently smooth.

(i) Then there exists a fine decoupling sequence $Q_{0}, \ldots, Q_{\mu-1}$.

(ii) The solution space $S_{\text {can } \mu}(t) \subseteq \mathbb{R}^{m}$ has constant dimension $d=(1-\mu) m+\sum_{i=0}^{\mu-1} r_{i}$ on $\mathcal{I}$.

(iii) The inherent regular explicit ODE (2.10) is independent of the special choice of the fine decoupling sequences, and so is its characteristic invariant (time-varying) subspace $i m D P_{0} \cdots P_{\mu-1} D^{-}$.

Proof: It remains to show (ii) and (iii).

(ii) is a consequence of the decomposition (2.16), which becomes true for fine decouplings.

(iii) Let $Q_{0}, \ldots, Q_{\mu-1}$ and $\bar{Q}_{0}, \ldots, \bar{Q}_{\mu-1}$ be admissible and both provide fine decouplings. Then, $\Pi_{\text {can } \mu}=\left(I-\mathcal{K}_{0}\right) P_{0} \cdots P_{\mu-1}=\left(I-\overline{\mathcal{K}}_{0}\right) \bar{P}_{0} \cdots \bar{P}_{\mu-1}$ is the projector onto $S_{\text {can } \mu}$ along $N_{\text {can } \mu}=$ $N_{0} \oplus \cdots \oplus N_{\mu-1}$. Taking into account that $\bar{D}^{-}=\bar{P}_{0} D^{-}, \Pi_{c a n \mu}=\Pi_{c a n \mu} P_{0}=\Pi_{c a n \mu} \bar{P}_{0}$ we find

$$
\begin{aligned}
D P_{0} \cdots P_{\mu-1} D^{-} & =D \Pi_{c a n \mu} D^{-}=D \Pi_{c a n \mu} \bar{P}_{0} D^{-}=D \Pi_{c a n \mu} \bar{D}^{-} \\
& =D \bar{P}_{0} \cdots \bar{P}_{\mu-1} \bar{D}^{-} \\
D P_{0} \cdots P_{\mu-1} G_{\mu}^{-1} & =D P_{0} \cdots P_{\mu-1} D^{-} D G_{\mu}^{-1}=D \bar{P}_{0} \cdots \bar{P}_{\mu-1} \bar{D}^{-} D Z_{\mu} \bar{G}_{\mu}^{-1} \\
& =D \bar{P}_{0} \cdots \bar{P}_{\mu-1} \bar{G}_{\mu}^{-1} \\
D P_{0} \cdots P_{\mu-1} G_{\mu}^{-1} B D^{-} & =D \bar{P}_{0} \cdots \bar{P}_{\mu-1} \bar{G}_{\mu}^{-1} B\left(\bar{P}_{0}+\bar{Q}_{0}\right) D^{-} \\
& =D \bar{P}_{0} \cdots \bar{P}_{\mu-1} \bar{G}_{\mu}^{-1} \bar{G}_{\mu} \bar{Q}_{0} D^{-}+D \bar{P}_{0} \cdots \bar{P}_{\mu-1} \bar{G}_{\mu}^{-1} B \bar{P}_{0} D^{-} \\
& =D \bar{P}_{0} \cdots \bar{P}_{\mu-1} \bar{G}_{\mu}^{-1} B \bar{D}^{-},
\end{aligned}
$$

hence, all coefficients of the explicit ODE (2.10) coincide, and $i m D P_{0} \cdots P_{\mu-1} D^{-}=i m D \bar{P}_{0} \cdots \bar{P}_{\mu-1} \bar{D}^{-}$.

Let us stress once again that, basically, we deal with continuous coefficients $A, D, B$ in (2.1). The additional smoothness demand in Theorem 4.3 concerns consecutive decompositions of $i m D$ into further subspaces spanned by continuously differentiable functions. This can be connected with special smoothness requirements to the coefficients $A, D, B$. Simultaneously, we have a close relation to the solvability of the DAE. A precise definition of these smoothness requirements would be highly technical, so that we do without it and content ourselves with the flat formulation sufficiently smooth. However, let us mention that the coefficients $A, D, B$ themselves are not differentiated. 


\section{Complete decouplings and standard canonical form}

In case of regular index-1 DAEs, the projector function $Q_{0}$ chosen in such a way that $k e r Q_{0}=S_{0}$, $S_{0}:=\left\{z \in \mathbb{R}^{m}: B_{0} z \in i m G_{0}\right\}$ provides the completely decoupled system (cf. [3])

$$
\begin{aligned}
u^{\prime}-R^{\prime}+D P_{0} G_{1}^{-1} B_{0} D^{-} u & =D P_{0} G_{1}^{-1} q, \\
v_{0} & =Q_{0} G_{1}^{-1} q
\end{aligned}
$$

since $Q_{0}=Q_{0} G_{1}^{-1} B_{0}$, and $N_{0} \oplus S_{0}=\mathbb{R}^{m}$.

In [3], projectors $Q_{0}, Q_{1}$ providing a complete decoupling of regular index-2 DAEs are described. Now we are able to generalize these results.

Theorem 5.1 Given a regular DAE with tractabilty index $\mu$ and fine decoupling projector functions $Q_{0}, \ldots Q_{\mu-1}$. Then there are admissible projector functions $\bar{Q}_{0}, \ldots \bar{Q}_{\mu-1}$ that provide a complete decoupling.

Proof: We choose $\bar{Q}_{0}:=\bar{Q}_{0 *}, \quad \bar{Q}_{i}:=Z_{i}^{-1} Q_{i} Z_{i}, \quad i=1, \ldots, \mu-1$. Taking into consideration that $\bar{D}^{-}=\bar{P}_{0} D^{-}, \bar{Q}_{0} Z_{i}^{-1}=\bar{Q}_{0}\left(I-\bar{Q}_{0} P_{0}\right)=Q_{0}$, we derive $\bar{Q}_{i} \bar{Q}_{j}=0$, for $j<i$, $\bar{Q}_{i} P_{i}=0$, for $i=1, \ldots, \mu-1$, further $\bar{P}_{0} \cdots \bar{P}_{i}=P_{0} \cdots P_{i}+Q_{0} \bar{P}_{0} P_{0} \cdots P_{i}=P_{0} \cdots P_{i}-\bar{Q}_{0} P_{0} \cdots P_{i}$, $D \bar{P}_{0} \cdots \bar{P}_{i} \bar{D}^{-}=D \bar{P}_{0} \cdots \bar{P}_{i} D^{-}=D P_{0} \cdots P_{i} D^{-}, i=1, \ldots, \mu-1$. Hence, the new projectors are admissible. Then we check $\bar{K}_{0}=\left(Q_{0 *}-\bar{Q}_{0}\right) P_{0} \cdots P_{\mu-1}, \bar{K}_{i}=0, i=1, \ldots, \mu-1$, analogously to Lemma 4.2 (cf. Appendix).

Each of the decoupled system equations (2.10) - (2.12) is formulated in $\mathbb{R}^{m}$, so that (2.10) - (2.12) is in $\mathbb{R}^{m(\mu+1)}$. In fact, there is a lower number equation so that we can condense the whole system to an equation in $\mathbb{R}^{m}$. We will show that the condensed system has standard canonical form (SCF), that is, it consists of the two parts (cf. [4])

$$
\begin{aligned}
y^{\prime}+M y & =L_{y} q, \\
N w^{\prime}+w & =L_{w} q,
\end{aligned}
$$

where $N$ is strictly upper triangular independent of $t$.

Let $Q_{0}, \ldots, Q_{\mu-1}$ be admissible projectors providing a complete decoupling (2.10) - (2.12), $\mathcal{K}_{i}=0, i=0,1, \ldots, \mu-1$. Then, the projector functions $D P_{0} \cdots P_{\mu-1} D^{-}, D P_{0} \cdots P_{\mu-2} Q_{\mu-1} D^{-}, \ldots$ $\ldots, D P_{0} Q_{1} D^{-}$are continuously differentiable and have $\operatorname{rank} d=(1-\mu) m+\sum_{i=0}^{\mu-1} r_{i}, m-r_{\mu-1}, \ldots, m-$ $r_{1}$, respectively. There are full rank continuously differentiable matrix functions $\mathcal{W}_{d}, \mathcal{W}_{\mu-1}, \ldots, \mathcal{W}_{1}$ (with $d, m-r_{\mu-1}, \ldots, m-r_{1}$ rows, but all with $m$ columns) such that $\mathcal{W}_{d}\left(i m D P_{0} \cdots P_{\mu-1} D^{-}\right)=$ $\mathbb{R}^{d}, \mathcal{W}_{i}\left(i m D P_{0} \cdots P_{i-1} Q_{i} D^{-}\right)=\mathbb{R}^{m-r_{i}}, i=1, \ldots, \mu-1$. The Moore-Penrose inverses $\mathcal{W}_{d}^{+}, \mathcal{W}_{i}^{+}$, $i=1, \ldots, \mu-1$, are also continuously differentiable. It holds that $\mathcal{W}_{i} \mathcal{W}_{i}^{+}=I$, and $\mathcal{W}_{i}^{+} \mathcal{W}_{i}$ is the orthoprojector function onto $i m D P_{0} \cdots P_{i-1} Q_{i} D^{-}, i=1, \ldots, \mu-1$. Analogously, $\mathcal{W}_{d} \mathcal{W}_{d}^{+}=$ $I, \mathcal{W}_{d}^{+} \mathcal{W}_{d}$ projects onto im $D P_{0} \cdots P_{\mu-1} D^{-}$.

Since $Q_{0}$ is continuous, there is a $\mathcal{W}_{0} \in C\left(\mathcal{I}, L\left(\mathbb{R}^{m}, \mathbb{R}^{m-r_{0}}\right)\right)$ such that $W_{0}\left(N_{0}\right)=\mathbb{R}^{m-r_{0}}$, $\mathcal{W}_{0} \mathcal{W}_{0}^{+}=I, \mathcal{W}_{0}^{+} \mathcal{W}_{0}$ projects onto $N_{0}$.

Multiplication of equation (2.10) by $\mathcal{W}_{d}$ yields, for $y=\mathcal{W}_{d} u$,

$$
y^{\prime}-\mathcal{W}_{d}^{\prime} \mathcal{W}_{d}^{+} y+\mathcal{W}_{d} D P_{0} \cdots P_{\mu-1} G_{\mu}^{-1} B D^{-} \mathcal{W}_{d}^{+} y=\mathcal{W}_{d} D P_{0} \cdots P_{\mu-1} G_{\mu}^{-1} q
$$

Here, due to $u=D P_{0} \cdots P_{\mu-1} D^{-} u$, we have $u=\mathcal{W}_{d}^{+} \mathcal{W}_{d} u=\mathcal{W}_{d}^{+} y$.

With $L_{y}:=\mathcal{W}_{d} D P_{0} \cdots P_{\mu-1} G_{\mu}^{-1}, M:=\mathcal{W}_{d} D P_{0} \cdots P_{\mu-1} G_{\mu}^{-1} B D^{-} \mathcal{W}_{d}^{+}-\mathcal{W}_{d}^{\prime} \mathcal{W}_{d}^{+}$we arrive at (5.3), 
i.e., an explicit ODE in $\mathbb{R}^{d} . y$ and the original unknown function $x$ are related by

$$
y=\mathcal{W}_{d} D P_{0} \cdots P_{\mu-1} x
$$

Next we multiply $(2.11)$ by $\mathcal{W}_{\mu-1} D$ and obtain, for $w_{\mu-1}:=\mathcal{W}_{\mu-1} D v_{\mu-1}$,

$$
w_{\mu-1}=\mathcal{W}_{\mu-1} D \mathcal{L}_{\mu-1} q .
$$

Due to $D v_{\mu-1}=D P_{0} \cdots P_{\mu-2} Q_{\mu-1} D^{-} D v_{\mu-1}$ it holds that $D v_{\mu-1}=\mathcal{W}_{\mu-1}^{+} \mathcal{W}_{\mu-1} D v_{\mu-1}=\mathcal{W}_{\mu-1}^{+} w_{\mu-1}$, further $v_{\mu-1}=D^{-} D v_{\mu-1}=D^{-} \mathcal{W}_{\mu-1}^{+} w_{\mu-1}$.

Analogously, for $k=1, \ldots, \mu-2$, put $w_{k}:=\mathcal{W}_{k} D v_{k}$ so that $v_{k}=D^{-} \mathcal{W}_{k}^{+} w_{k}, D v_{k}=\mathcal{W}_{k}^{+} w_{k}$, and (2.12) yields

$$
w_{k}=\sum_{j=k+1}^{\mu-1} \mathcal{W}_{k} D \mathcal{N}_{k j}\left(\mathcal{W}_{j}^{+\prime} w_{j}+\mathcal{W}_{j}^{+} w_{j}^{\prime}\right)+\sum_{j=k+2}^{\mu-1} \mathcal{W}_{k} D \mathcal{M}_{k j} D^{-} \mathcal{W}_{j}^{+} w_{j}+\mathcal{W}_{k} D \mathcal{L}_{k} q
$$

i.e.,

$$
w_{k}=\sum_{j=k+1}^{\mu-1} \widetilde{\mathcal{N}}_{k j} w_{j}^{\prime}+\sum_{j=k+1}^{\mu-1} \widetilde{\mathcal{M}}_{k j} w_{j}+\mathcal{W}_{k} D \mathcal{L}_{k} q, k=1, \ldots, \mu-2 .
$$

The coefficients $\widetilde{\mathcal{N}}_{k j}, \widetilde{\mathcal{M}}_{k j}$ are continuous.

Finally, equation (2.12) with $k=0$, leads to

$$
\begin{gathered}
w_{0}:=\mathcal{W}_{0} v_{0}, \quad v_{0}=\mathcal{W}_{0}^{+} w_{0}, \\
w_{0}=\sum_{j=1}^{\mu-1} \widetilde{\mathcal{N}}_{0 j} w_{j}^{\prime}+\sum_{j=1}^{\mu-1} \widetilde{\mathcal{M}}_{0 j} w_{j}+\mathcal{W}_{0} \mathcal{L}_{0} q .
\end{gathered}
$$

Collect (5.7), (5.6) and (5.5) together to

$$
\begin{aligned}
\left(\begin{array}{c}
w_{0} \\
w_{1} \\
\vdots \\
w_{\mu-1}
\end{array}\right)=\underbrace{\left(\begin{array}{cccc}
0 & \widetilde{\mathcal{N}}_{01} & \cdots & \widetilde{\mathcal{N}}_{0 \mu-1} \\
0 & 0 & \ddots & \vdots \\
\vdots & \vdots & \ddots & \widetilde{\mathcal{N}}_{\mu-2 \mu-1} \\
0 & 0 & \cdots & 0
\end{array}\right)}_{\widetilde{\mathcal{N}}}\left(\begin{array}{c}
0 \\
w_{1}^{\prime} \\
\vdots \\
w_{\mu-1}
\end{array}\right)+\underbrace{\left(\begin{array}{cccc}
0 & \widetilde{\mathcal{M}}_{01} & \cdots & \widetilde{\mathcal{M}}_{0 \mu-1} \\
\vdots & \ddots & \ddots & \vdots \\
\vdots & \vdots & \ddots & \widetilde{\mathcal{M}}_{\mu-2 \mu-1} \\
0 & \cdots & \cdots & 0
\end{array}\right)}_{\widetilde{\mathcal{M}}} \\
+\left(\begin{array}{c}
\mathcal{W}_{0} \mathcal{L}_{0} q \\
\mathcal{W}_{1} D \mathcal{L}_{1} q \\
\vdots \\
\mathcal{W}_{\mu-1} D \mathcal{L}_{\mu-1} q
\end{array}\right)
\end{aligned}
$$

Finally, scaling the last system by the inverse of $I-\widetilde{\mathcal{M}}$, we obtain the system

$$
\left(\begin{array}{c}
w_{0} \\
w_{1} \\
\vdots \\
w_{\mu-1}
\end{array}\right)+\mathcal{N}\left(\begin{array}{c}
0 \\
w_{1}^{\prime} \\
\vdots \\
w_{\mu-1}^{\prime}
\end{array}\right)=L_{w} q
$$


where $\mathcal{N}:=-(I-\widetilde{\mathcal{M}})^{-1} \widetilde{\mathcal{N}}$ is strictly block upper triangular, $\mathcal{N}^{\mu}=0$, and

$L_{w}:=(I-\widetilde{\mathcal{M}})^{-1}\left(\begin{array}{c}\mathcal{W}_{0} \mathcal{L}_{0} \\ \mathcal{W}_{1} D \mathcal{L}_{1} \\ \vdots \\ \mathcal{W}_{\mu-1} D \mathcal{L}_{\mu-1}\end{array}\right)$. All coefficients are continuous. System (5.8) consists of $\mu m-$ $\sum_{t=0}^{\mu-1} r_{i}$ equations. By these considerations the following statement is proved.

Theorem 5.2 A regular DAE with sufficiently smooth coefficients is equivalent to a DAE in SCF.

Supposed the function $q$, the coefficient $L_{w}$, and the entries of $\mathcal{N}$ in (5.8) are smooth enough, the differentiations can be carried out. By this, equation (5.8) is replaced by the simple assessment

$$
w=g
$$

where $g$ now contains all resulting derivatives. On the other hand, transforming $y=T z$ in (5.3), with $T$ being a fundamental solution matrix to the ODE $y^{\prime}+M y=0$, yields the trivial ODE

$$
z^{\prime}=f
$$

It turns out that a regular DAE with tractability index $\mu$, the characteristic numbers $r_{0}, \ldots, r_{\mu-1}$, and sufficiently smooth coefficients, is equivalent to a DAE in so-called (cf. [8]) strangeness-free form (5.9),(5.10), with $z(t) \in \mathbb{R}^{d}, w_{i}(t) \in \mathbb{R}^{m-r_{i}}, i=0, \ldots, \mu-1$.

We point out that both, the SCF (5.3),(5.8) and the strangeness-free form DAE (5.9),(5.10) are given via transformations of the unknown variable while the decoupled system $(2.10),(2.11),(2.12)$ is given in the original setting of the DAE.

\section{Concluding remark}

It is shown that, under certain smoothness conditions concerning some special subspaces, each regular DAE has both, fine and complete decouplings. Moreover, each such DAE is equivalent to a DAE in SCF.

Basically, the DAE coefficients are only continuous. The additional smoothness attached to the demand for the existence of admissible projector functions providing a fine decoupling is closely related to solvability.

Roughly speaking, one can say that a solvable regular DAE is equivalent to a DAE in SCF. This sounds very similar to results in [4], however, the understanding of solvability is quite different.

Fine decouplings are an appropriate tool for the further analysis of DAEs. Since there is an inherent explicit ODE that is uniquely determined by the problem data, the qualitative behaviour of the flow can be studied. Also, strong solvability statements are available (cf. [1]), in particular the function spaces of admissible right-hand sides $q$ leading to $C_{D}^{1}$ solutions can be described precisely. If $q$ has lower regularity, the decoupled system shows clearly which components suffer from the discontinuities etc.

\section{Appendix: Proof of Lemma 4.2}

Given admissible projector functions $Q_{0}, \ldots, Q_{\mu-1}$ for the regular DAE (2.1) with index $\mu$, with $Q_{j *} P_{0} \cdots P_{\mu-1}=0$, for $j=k+1, \ldots, \mu-1$, and $k \in\{2, \ldots, \mu-2\}$ fixed. 
Put $\bar{Q}_{j}:=Q_{j}, j=0, \ldots, k-1$ and let $\bar{Q}_{k}$ be such that $\bar{Q}_{0}, \ldots, \bar{Q}_{k}$ is admissible up to order $k$. Put further $\bar{Q}_{j}:=Z_{j}^{-1} Q_{j} Z_{j}=Q_{j}, j=k+1, \ldots, \mu-1$. Then $\bar{Q}_{0}, \ldots, \bar{Q}_{\mu-1}$ are admissible. It holds that $\bar{G}_{j}=G_{j} Z_{j}, j=0, \ldots, \mu ; Z_{j}=I, j=0, \ldots, k$,

$$
\begin{aligned}
Z_{k+1} & =I+\bar{Q}_{k} P_{k}+\sum_{j=0}^{k-1} Q_{j} Z_{k j} \bar{Q}_{k}, \\
\bar{Q}_{k} P_{k} & =-Q_{k} \bar{P}_{k}, \\
\bar{Q}_{i} P_{i} & =0, \quad i=k+1, \ldots, \mu-1, \\
Z_{i+1} & =\left(I+\sum_{j=0}^{i-1} Q_{j} Z_{i j} \bar{Q}_{i}\right) Z_{i}, \quad i=k+1, \ldots, \mu-1 .
\end{aligned}
$$

Further, we have for $i \geq k+1$

$$
\begin{gathered}
\bar{P}_{0} \cdots \bar{P}_{i}=P_{0} \cdots P_{k-1} \bar{P}_{k} P_{k+1} \cdots P_{i} \text { and } \\
D \bar{P}_{0} \cdots \bar{P}_{i} D^{-}=D P_{0} \cdots P_{i} D^{-}-D P_{0} \cdots P_{k-1} \bar{Q}_{k} D^{-} \cdot D P_{0} \cdots P_{i} D^{-} .
\end{gathered}
$$

Compute

$$
\begin{array}{r}
\bar{Q}_{k *} \bar{P}_{0} \cdots \bar{P}_{\mu-1}=\bar{Q}_{k} \bar{P}_{k+1} \cdots \bar{P}_{\mu-1} \bar{G}_{\mu}^{-1}\left\{\bar{B}_{k}+\bar{G}_{k} D^{-}\left(D \bar{P}_{0} \cdots \bar{P}_{\mu-1} D^{-}\right)^{\prime} D \bar{P}_{0} \cdots \bar{P}_{k-1}\right\} \bar{P}_{0} \cdots \bar{P}_{\mu-1} \\
=\bar{Q}_{k} \bar{P}_{k+1} \cdots \bar{P}_{\mu-1} \bar{G}_{\mu}^{-1}\left\{B_{0} P_{0} \cdots P_{k-1} \bar{P}_{k} P_{k+1} \cdots P_{\mu-1}+\right. \\
\left.+G_{k} D^{-}\left(D P_{0} \cdots \bar{P}_{k} \cdots P_{\mu-1} D^{-}\right)^{\prime} D P_{0} \cdots \bar{P}_{k} \cdots P_{\mu-1}\right\}
\end{array}
$$

and with $\mathfrak{B}:=B_{0} P_{0} \cdots P_{k-1} \bar{P}_{k} P_{k+1} \cdots P_{\mu-1}+G_{k} D^{-}\left(D P_{0} \cdots \bar{P}_{k} \cdots P_{\mu-1} D^{-}\right)^{\prime} D P_{0} \cdots \bar{P}_{k} \cdots P_{\mu-1}$, taking into account that

$$
\bar{Q}_{k} Z_{k+1}^{-1}=\bar{Q}_{k}\left(I-\bar{Q}_{k} P_{k}\right)=Q_{k}
$$

we obtain

$$
\begin{aligned}
\bar{Q}_{k *} \bar{P}_{0} \cdots & \bar{P}_{\mu-1}=Q_{k} P_{k+1} Z_{k+1} Z_{k+2}^{-1} P_{k+2} \cdots Z_{\mu-2} Z_{\mu-1}^{-1} P_{\mu-1} Z_{\mu-1} Z_{\mu}^{-1} G_{\mu}^{-1} \mathfrak{B} \\
= & Q_{k} P_{k+1}\left(I-Q_{k} Z_{k+1 k} Q_{k+1}\right) P_{k+2} \cdots \\
& \cdots\left(I-Q_{\mu-3} Z_{\mu-2 \mu-3} Q_{\mu-2}\right) P_{\mu-1}\left(I-Q_{\mu-2} Z_{\mu-1 \mu-2} Q_{\mu-1}\right) G_{\mu}^{-1} \mathfrak{B} \\
= & Q_{k} P_{k+1} \cdots P_{\mu-1} G_{\mu}^{-1} \mathfrak{B}+\mathfrak{A}_{k+1} Q_{k+1} P_{k+2} \cdots P_{\mu-1} G_{\mu}^{-1} \mathfrak{B}+\cdots+\mathfrak{A}_{\mu-1} Q_{\mu-1} G_{\mu}^{-1} \mathfrak{B} .
\end{aligned}
$$

Next we show that the terms $Q_{\mu-1} G_{\mu}^{-1} \mathfrak{B}, \ldots, Q_{k+1} P_{k+2} \cdots P_{\mu-1} G_{\mu}^{-1} \mathfrak{B}$ in (7.2) disappear. For this purpose we reformualte $\mathfrak{B}$ as follows

$$
\begin{aligned}
\mathfrak{B}= & B_{0} P_{0} \cdots P_{\mu-1}+G_{k} D^{-}\left(D P_{0} \cdots P_{\mu-1} D^{-}\right)^{\prime} D P_{0} \cdots P_{\mu-1} \\
& +B_{0} P_{0} \cdots P_{k-1} Q_{k} \bar{P}_{k} P_{0} \cdots P_{\mu-1}+G_{k} D^{-}\left(D P_{0} \cdots P_{\mu-1} D^{-}\right)^{\prime} D P_{0} \cdots P_{k-1} Q_{k} \bar{P}_{k} P_{0} \cdots P_{\mu-1} \\
& +G_{k} D^{-}\left(D P_{0} \cdots P_{k-1} Q_{k} \bar{P}_{k} P_{0} \cdots P_{\mu-1} D^{-}\right) D P_{0} \cdots P_{k-1} \bar{P}_{k} P_{k+1} \cdots P_{\mu-1} \\
= & B_{0} P_{0} \cdots P_{\mu-1}+G_{k} D^{-}\left(D P_{0} \cdots P_{\mu-1} D^{-}\right)^{\prime} D P_{0} \cdots P_{\mu-1} \\
& +\left(B_{k}+G_{k} D^{-}\left(D P_{0} \cdots P_{\mu-1} D^{-}\right)^{\prime} D P_{0} \cdots P_{k-1}\right) Q_{k} \bar{P}_{k} P_{0} \cdots P_{\mu-1} \\
& +\sum_{j=1}^{k} G_{j} D^{-}\left(D P_{0} \cdots P_{j} D^{-}\right)^{\prime} D P_{0} \cdots P_{k-1} Q_{k} \bar{P}_{k} P_{0} \cdots P_{\mu-1} \\
& +G_{k} D^{-}\left(D P_{0} \cdots P_{k-1} Q_{k} \bar{P}_{k} P_{0} \cdots P_{\mu-1} D^{-}\right)^{\prime} D P_{0} \cdots P_{k-1} \bar{P}_{k} P_{k+1} \cdots P_{\mu-1} .
\end{aligned}
$$


Since $Q_{\mu-1} G_{\mu}^{-1} G_{j}=Q_{\mu-1} P_{\mu-1} \cdots P_{j}=0$, for $j \leq \mu-1$, and $Q_{\mu-1} G_{\mu}^{-1} B_{k} Q_{k}=Q_{\mu-1} Q_{k}=0$, we find immediately

$$
Q_{\mu-1} G_{\mu}^{-1} \mathfrak{B}=Q_{\mu-1} G_{\mu}^{-1} B_{0} P_{0} \cdots P_{\mu-1}=Q_{\mu-1 *} P_{0} \cdots P_{\mu-1}=0
$$

For $i=k+1, \ldots, \mu-2$, it holds that

$$
\begin{aligned}
& Q_{i} P_{i+1} \cdots P_{\mu-1} G_{\mu}^{-1}\left\{G_{k}-G_{i}\right\}=-Q_{i} P_{i+1} \cdots P_{\mu-1} G_{\mu}^{-1}\left\{B_{k} Q_{k}+\cdots+B_{i-1} Q_{i-1}\right\}=0 \\
& Q_{i} P_{i+1} \cdots P_{\mu-1} G_{\mu}^{-1} B_{k} Q_{k}=0 \\
& Q_{i} P_{i+1} \cdots P_{\mu-1} G_{\mu}^{-1} G_{k} D^{-}\left(D P_{0} \cdots P_{\mu-1} D^{-}\right)^{\prime} D P_{0} \cdots P_{k-1} Q_{k} \\
& \quad=Q_{i} P_{i+1} \cdots P_{\mu-1} P_{i} D^{-}\left(D P_{0} \cdots P_{\mu-1} D^{-}\right)^{\prime} D P_{0} \cdots P_{k-1} Q_{k} \\
& \quad=-Q_{i} P_{i+1} \cdots P_{\mu-1} P_{i} \cdot P_{0} \cdots P_{\mu-1} D^{-}\left(D P_{0} \cdots P_{k-1} Q_{k} D^{-}\right)^{\prime} D=0
\end{aligned}
$$

and

$$
\begin{aligned}
& Q_{i} P_{i+1} \cdots P_{\mu-1} G_{\mu}^{-1} \sum_{j=1}^{k} G_{j} D^{-}\left(D P_{0} \cdots P_{j} D^{-}\right)^{\prime} D P_{0} \cdots P_{k-1} Q_{k} \bar{P}_{k} P_{0} \cdots P_{\mu-1} \\
& =Q_{i} P_{i+1} \cdots P_{\mu-1} P_{i} D^{-}\left(D P_{0} \cdots P_{k} D^{-}\right)^{\prime} D P_{0} \cdots P_{k-1} Q_{k} \bar{P}_{k} P_{0} \cdots P_{\mu-1} .
\end{aligned}
$$

This yields, for $i=k+1, \ldots, \mu-2$,

$$
\begin{aligned}
& Q_{i} P_{i+1} \cdots P_{\mu-1} G_{\mu}^{-1} \mathfrak{B}=Q_{i *} P_{i+1} \cdots P_{\mu-1}+ \\
& \quad+Q_{i} P_{i+1} \cdots P_{\mu-1} P_{i} D^{-}\left(D P_{0} \cdots P_{k} D^{-}\right)^{\prime} D P_{0} \cdots P_{k-1} Q_{k} \bar{P}_{k} P_{0} \cdots P_{\mu-1} \\
& \quad+Q_{i} P_{i+1} \cdots P_{\mu-1} P_{i} D^{-}\left(D P_{0} \cdots P_{k-1} Q_{k} \bar{P}_{k} P_{0} \cdots P_{\mu-1} D^{-}\right)^{\prime} D P_{0} \cdots P_{k-1} \bar{P}_{k} P_{k+1} \cdots P_{\mu-1},
\end{aligned}
$$

and because of

$$
\begin{aligned}
& Q_{i} P_{i+1} \cdots P_{\mu-1} P_{i} D^{-}\left(D P_{0} \cdots P_{k-1} Q_{k} \bar{P}_{k} P_{0} \cdots P_{\mu-1} D^{-}\right)^{\prime} D P_{0} \cdots P_{k-1} \bar{P}_{k} P_{k+1} \cdots P_{\mu-1} \\
& \quad=-Q_{i} P_{i+1} \cdots P_{\mu-1} P_{i} D^{-}\left(D P_{0} \cdots P_{k} D^{-}\right)^{\prime} D P_{0} \cdots P_{k-1} Q_{k} \bar{P}_{k} P_{0} \cdots P_{\mu-1}
\end{aligned}
$$

we obtain from $(7.5)$

$$
Q_{i} P_{i+1} \cdots P_{\mu-1} G_{\mu}^{-1} \mathfrak{B}=Q_{i *} P_{i+1} \cdots P_{\mu-1}=0
$$

Now, (7.2) simplifies to

$$
\bar{Q}_{i *} \bar{P}_{0} \cdots \bar{P}_{\mu-1}=Q_{k} P_{k+1} \cdots P_{\mu-1} G_{\mu}^{-1} \mathfrak{B} .
$$

Because of $Q_{k} P_{k+1} \cdots P_{\mu-1} G_{\mu}^{-1} B_{k} Q_{k}=Q_{k}$,

$$
\begin{aligned}
& Q_{k} P_{k+1} \cdots P_{\mu-1} G_{\mu}^{-1} G_{k} D^{-}\left(D P_{0} \cdots P_{\mu-1} D^{-}\right)^{\prime} D P_{0} \cdots P_{k-1} Q_{k} \\
& =-Q_{k} P_{k+1} \cdots P_{\mu-1} P_{k} P_{0} \cdots P_{\mu-1} D^{-}\left({ }^{\prime} D P_{0} \cdots P_{k-1} Q_{k} D^{-}\right) D=0 \\
& Q_{k} P_{k+1} \cdots P_{\mu-1} G_{\mu}^{-1} \sum_{j=1}^{k} G_{j} D^{-}\left(D P_{0} \cdots P_{j} D^{-}\right)^{\prime} D P_{0} \cdots P_{k-1} Q_{k} \bar{P}_{k} P_{0} \cdots P_{\mu-1} \\
& =Q_{k} P_{k+1} \cdots P_{\mu-1} P_{k} D^{-}\left(D P_{0} \cdots P_{k} D^{-}\right)^{\prime} D P_{0} \cdots P_{k-1} Q_{k} \bar{P}_{k} P_{0} \cdots P_{\mu-1}
\end{aligned}
$$

and (cf. (7.3))

$$
\begin{aligned}
& Q_{k} P_{k+1} \cdots P_{\mu-1} G_{\mu}^{-1} G_{k} D^{-}\left(D P_{0} \cdots P_{k-1} Q_{k} \bar{P}_{k} P_{0} \cdots P_{\mu-1} D^{-}\right)^{\prime} D P_{0} \cdots P_{k-1} \bar{P}_{k} P_{k+1} \cdots P_{\mu-1} \\
& =-Q_{k} P_{k+1} \cdots P_{\mu-1} P_{k} D^{-}\left(D P_{0} \cdots P_{k} D^{-}\right)^{\prime} D P_{0} \cdots P_{k-1} Q_{k} \bar{P}_{k} P_{0} \cdots P_{\mu-1}
\end{aligned}
$$


the relation $Q_{k} P_{k+1} \cdots P_{\mu-1} G_{\mu}^{-1} \mathfrak{B}=Q_{k *} P_{0} \cdots P_{\mu-1}+Q_{k} \bar{P}_{k} P_{0} \cdots P_{\mu-1}=Q_{k *} P_{0} \cdots P_{\mu-1}-\bar{Q}_{k} P_{0} \cdots$ $\cdots P_{\mu-1}$, i.e., (cf. (7.7)) $\bar{Q}_{\mu *} \bar{P}_{0} \cdots \bar{P}_{\mu-1}=\left(Q_{k *}-\bar{Q}_{k}\right) P_{0} \cdots P_{\mu-1}$ follows. By this, relation (4.12) in Lemma 4.2 is verified.

Now we turn to relation (4.11). Obviously, it holds that

$$
\begin{aligned}
& \bar{Q}_{\mu-1 *} \bar{P}_{0} \cdots \bar{P}_{\mu-1}=\bar{Q}_{\mu-1} \bar{G}_{\mu}^{-1} B_{0} \bar{P}_{0} \cdots \bar{P}_{\mu-1}=\bar{Q}_{\mu-1} \bar{G}_{\mu}^{-1} B_{0} \bar{P}_{\mu-1} \\
& =Z_{\mu-1}^{-1} Q_{\mu-1} Z_{\mu-1} Z_{\mu}^{-1} G_{\mu}^{-1} B_{0} Z_{\mu-1}^{-1} P_{\mu-1} Z_{\mu-1}=Z_{\mu-1}^{-1} Q_{\mu-1} G_{\mu}^{-1} B_{0} P_{0} \cdots P_{\mu-1} \\
& =Z_{\mu-1}^{-1} Q_{\mu-1 *} P_{0} \cdots P_{\mu-1}=0
\end{aligned}
$$

For $i=k+1, \ldots, \mu-2$ we derive

$$
\begin{gathered}
\bar{Q}_{i *} \bar{P}_{0} \cdots \bar{P}_{\mu-1}=\bar{Q}_{i} \bar{P}_{i+1} \cdots \bar{P}_{\mu-1} \bar{G}_{\mu}^{-1}\left\{B_{0} \bar{P}_{0} \cdots \bar{P}_{\mu-1}+\bar{G}_{i} D^{-}\left(D \bar{P}_{0} \cdots \bar{P}_{\mu-1} D^{-}\right)^{\prime} D \bar{P}_{0} \cdots \bar{P}_{\mu-1}\right\} \\
=Z_{i}^{-1} Q_{i} Z_{i} Z_{i+1}^{-1} P_{i+1} \cdots Z_{\mu-2} Z_{\mu-1}^{-1} P_{\mu-1} Z_{\mu-1} Z_{\mu}^{-1} G_{\mu}^{-1} \cdot \mathcal{D}_{i} \\
=Z_{i}^{-1} Q_{i} P_{i+1}\left(I-Q_{i} Z_{i+1 i} Q_{i+1}\right) P_{i+2} \cdots\left(I-Q_{\mu-3} Z_{\mu-2 \mu-3} Q_{\mu-2}\right) P_{\mu-1} \cdot \\
\quad \cdot\left(I-Q_{\mu-2} Z_{\mu-1 \mu-2} Q_{\mu-1}\right) G_{\mu}^{-1} \mathcal{D}_{i} \\
=Z_{i}^{-1} Q_{i} P_{i+1} P_{i+2} \cdots P_{\mu-1} G_{\mu}^{-1} \mathcal{D}_{i}+E_{i+1} Q_{i+1} P_{i+2} \cdots P_{\mu-1} G_{\mu}^{-1} \mathcal{D}_{i} \\
\quad+\cdots+E_{\mu-2} Q_{\mu-2} P_{\mu-1} G_{\mu}^{-1} \mathcal{D}_{i}+E_{\mu-1} Q_{\mu-1} G_{\mu}^{-1} \mathcal{D}_{i},
\end{gathered}
$$

where

$$
\begin{aligned}
& \mathcal{D}_{i}:=B_{0} \bar{P}_{0} \cdots \bar{P}_{\mu-1}+\bar{G}_{i} D^{-}\left(D \bar{P}_{0} \cdots \bar{P}_{\mu-1} D^{-}\right)^{\prime} D \bar{P}_{0} \cdots \bar{P}_{\mu-1} \\
& =B_{0} P_{0} \cdots P_{\mu-1}+B_{0} P_{0} \cdots P_{k-1} Q_{k} \bar{P}_{k} P_{0} \cdots P_{\mu-1}+G_{i}\left(Z_{i}-I\right) D^{-}\left(D \bar{P}_{0} \cdots \bar{P}_{\mu-1} D^{-}\right)^{\prime} D \bar{P}_{0} \cdots \bar{P}_{\mu-1} \\
& +G_{i} D^{-}\left(D P_{0} \cdots P_{\mu-1} D^{-}\right)^{\prime} D P_{0} \cdots P_{\mu-1}+G_{i} D^{-}\left(\left(D P_{0} \cdots P_{\mu-1} D^{-}\right)^{\prime} D P_{0} \cdots P_{k-1} Q_{k} \bar{P}_{k} P_{0} \cdots P_{\mu-1}\right. \\
& +G_{i} D^{-}\left(D P_{0} \cdots P_{k-1} Q_{k} \bar{P}_{k} P_{0} \cdots P_{\mu-1} D^{-}\right)^{\prime} D P_{0} \cdots P_{k-1} \bar{P}_{k} P_{k+1} \cdots P_{\mu-1},
\end{aligned}
$$

hence,

$$
\begin{aligned}
\mathcal{D}_{i}= & B_{0} P_{0} \cdots P_{\mu-1}+G_{i} D^{-}\left(D P_{0} \cdots P_{\mu-1} D^{-}\right)^{\prime} D P_{0} \cdots P_{\mu-1} \\
& +G_{i}\left(Z_{i}-I\right) D^{-}\left(D \bar{P}_{0} \cdots \bar{P}_{\mu-1} D^{-}\right)^{\prime} D \bar{P}_{0} \cdots \bar{P}_{\mu-1} \\
& -G_{i} P_{0} \cdots P_{\mu-1} D^{-}\left(D P_{0} \cdots P_{k-1} Q_{k} D^{-}\right) D P_{0} \cdots P_{k-1} P_{k} P_{0} \cdots P_{\mu-1} \\
& +\left(B_{k}+\sum_{j=1}^{k} G_{j} D^{-}\left(D P_{0} \cdots P_{j} D^{-}\right) D P_{0} \cdots P_{k-1}\right) Q_{k} \bar{P}_{k} P_{0} \cdots P_{\mu-1} \\
& +G_{i} D^{-}\left(D P_{0} \cdots P_{k-1} Q_{k} \bar{P}_{k} P_{0} \cdots P_{\mu-1} D^{-}\right)^{\prime} D P_{0} \cdots P_{k-1} \bar{P}_{k} P_{k+1} \cdots P_{\mu-1} .
\end{aligned}
$$

Since $Q_{\mu-1} G_{\mu}^{-1} \bar{G}_{i}=Q_{\mu-1} G_{\mu}^{-1} G_{i} Z_{i}=Q_{\mu-1} P_{\mu-1} \cdots P_{i} Z_{i}=0$, one has $Q_{\mu-1} G_{\mu}^{-1} \mathcal{D}_{i}=$ $Q_{\mu-1} G_{\mu}^{-1} B_{0} \bar{P}_{0} \cdots \bar{P}_{\mu-1}=Q_{\mu-1} G_{\mu}^{-1} B_{0} P_{0} \cdots P_{\mu-2} Z_{\mu-1}^{-1} P_{\mu-1} Z_{\mu-1}=Q_{\mu-1} G_{\mu}^{-1} B_{0} P_{0} \cdots P_{\mu-1}=$ $Q_{\mu-1 *} P_{0} \cdots P_{\mu-1}=0$.

It remains to show that $Q_{l} P_{l+1} \cdots P_{\mu-1} G_{\mu}^{-1} \mathcal{D}_{i}$ vanishes for $l=i, \ldots, \mu-2$ and , consequently, $\bar{Q}_{i *} \bar{P}_{0} \cdots \bar{P}_{\mu-1}=0$.

We have $l \geq i \geq k+1$ and

$$
\begin{gathered}
Q_{l} P_{l+1} \cdots P_{\mu-1} G_{\mu}^{-1} G_{i}\left(Z_{i}-I\right)=Q_{l} P_{l+1} \cdots P_{\mu-1} P_{l} \sum_{j=0}^{i-1} Q_{j} Z_{i-1 j} Q_{i-1}=0 \\
Q_{l} P_{l+1} \cdots P_{\mu-1} G_{\mu}^{-1} G_{i} P_{0} \cdots P_{\mu-1}=Q_{l} P_{l+1} \cdots P_{\mu-1} P_{l} \cdot P_{0} \cdots P_{\mu-1}=0 \\
Q_{l} P_{l+1} \cdots P_{\mu-1} G_{\mu}^{-1} B_{k} Q_{k}=Q_{l} Q_{k}=0
\end{gathered}
$$




$$
\begin{aligned}
& Q_{l} P_{l+1} \cdots P_{\mu-1} G_{\mu}^{-1} \sum_{j=1}^{k} G_{j} D^{-}\left(D P_{0} \cdots P_{j} D^{-}\right)^{\prime} D P_{0} \cdots P_{k-1} Q_{k} \bar{P}_{k} P_{0} \cdots P_{\mu-1} \\
& =Q_{l} P_{l+1} \cdots P_{\mu-1} P_{l} D^{-}\left(D P_{0} \cdots P_{k} D^{-}\right)^{\prime} D P_{0} \cdots P_{k-1} Q_{k} \bar{P}_{k} P_{0} \cdots P_{\mu-1}, \\
& Q_{l} P_{l+1} \cdots P_{\mu-1} G_{\mu}^{-1} G_{i} D^{-}\left(D P_{0} \cdots P_{k-1} Q_{k} \bar{P}_{k} P_{0} \cdots P_{\mu-1} D^{-}\right)^{\prime} D P_{0} \cdots P_{k-1} \bar{P}_{k} P_{k+1} \cdots P_{\mu-1} \\
& =-Q_{l} P_{l+1} \cdots P_{\mu-1} P_{l} D^{-}\left(D P_{0} \cdots P_{k} D^{-}\right)^{\prime} D P_{0} \cdots P_{k-1} Q_{k} \bar{P}_{k} P_{0} \cdots P_{\mu-1} .
\end{aligned}
$$

Inserting these expressions (7.10) - (7.14) into $Q_{l} P_{l+1} \cdots P_{\mu-1} G_{\mu}^{-1} \mathcal{D}_{i}$ we find finally

$$
\begin{aligned}
Q_{l} P_{l+1} \cdots & P_{\mu-1} G_{\mu}^{-1} \mathcal{D}_{i}= \\
& =Q_{l} P_{l+1} \cdots P_{\mu-1} G_{\mu}^{-1}\left\{B_{0} P_{0} \cdots P_{\mu-1}+G_{i} D^{-}\left(D P_{0} \cdots P_{\mu-1} D^{-}\right)^{\prime} D P_{0} \cdots P_{\mu-1}\right\} \\
& =Q_{l} P_{l+1} \cdots P_{\mu-1} G_{\mu}^{-1}\left\{B_{0} P_{0} \cdots P_{\mu-1}+G_{l} D^{-}\left(D P_{0} \cdots P_{\mu-1} D^{-}\right)^{\prime} D P_{0} \cdots P_{\mu-1}\right\} \\
& =Q_{l *} P_{0} \cdots P_{\mu-1}=0 .
\end{aligned}
$$

\section{References}

[1] R. März: Solvability of linear differential algebraic equations with properly stated leading term. Results in Mathematics, to appear.

[2] R. März: The index of linear differential algebraic equations with properly stated leading term. Results in Mathematics 42(2003), 308-338.

[3] K. Balla und R. März: A unified approach to linear differential algebraic equations and their adjoints. Journal for Analysis and its Application 21(2002), 783-802.

[4] S.L. Campbell: A general form for solvable linear time varying singular systems of differential equations. SIAM J. Mat. Anal. 18(1987)4, 1101-1115.

[5] P.J. Rabier, W.C. Rheinboldt: Theoretical and numerical analysis of differential-algebraic equations. Handbook of Numerical Analysis VIII, Elsevier Publ., Amsterdam 2002.

[6] S.L. Campbell, L.R. Petzold: Canonical forms and solvable singular systems of differential equations. SIAM J. Alg. Discr. Methods 4(1983), 517-521.

[7] I. Schumilina: Index-3 DAEs with properly stated leading term. Humboldt-Universität zu Berlin, Institut für Mathematik, Preprint 2001-20, 2001.

[8] P. Kunkel, V. Mehrmann: Canonical forms for linear differential-algebraic equations with variable coefficients, J. Comput. Appl. Math. 56 (1994) 225-251. 